\title{
Prophylaxis of the Autoimmune Diseases
}

\author{
Duncan D. Adams ${ }^{1}$, John G. Knight ${ }^{2}$ and Alan Ebringer ${ }^{3}$ \\ 1. Faculty of Medicine, University of Otago, Dunedin, New Zealand \\ 2. Faculty of Commerce, University of Otago, Dunedin, New Zealand \\ 3. King's College, University of London, United Kingdom
}

\begin{abstract}
Pasteur's correct Germ Theory of Disease led to discovery of the Immunity System that exists for defense against diseases caused by bacteria or virus infections. In order to cope with the huge diversity of microbial infections, the immunity system needs a flexibility of immune response. This is provided by use of semi-random somatic gene mutations in lymphocytes, the cells that recognize invading bacteria and viruses. Burnet realized that this flexibility leads to development of the Forbidden Clones that cause the autoimmune diseases by accidentally being reactive with a host antigen instead of a microbial one. Ebringer has recently discovered the microbial triggers of rheumatoid arthritis and ankylosing spondylitis and has determined the amino acid sequences of the antigens on them that trigger the related autoimmune diseases. This has explained how histocompatibility antigens can predispose to autoimmune diseases. The Salk and Sabin anterior poliomyelitis vaccines have prevented the polio epidemic leg paralyses. We postulate that these paralyses were rare autoimmune complications of virtually universal poliovirus infection. As autoimmune diseases are triggered by microbial infections, we suggest that the triggering bacteria or viruses be sought, so that prophylaxis of the autoimmune diseases can be achieved by vaccination against their triggering microbes.
\end{abstract}

Key words: Forbidden Clone theory, H Gene theory, microbial triggers of autoimmune diseases, poliomyelitis vaccines, prophylaxis of poliomyelitis paralyses, prophylaxis of autoimmune diseases.

\section{Pathogenesis}

The long mysterious pathogenesis and genetics of the autoimmune diseases is now solved [1]. The key to the pathogenesis is Jerne's selection theory of antibody formation [2], which led to Burnet's clonal selection theory of acquired immunity [3] and his forbidden clone theory of autoimmune disease [4]. This states that somatic gene mutations in multiplying lymphocytes produce the appropriately-named Forbidden Clones that cause the autoimmune diseases by reacting with a host antigen instead of a microbial one.

After discovery that the thyroid gland over-activity of Graves' disease is caused by autoantibodies that react with the thyroid's receptor for thyroid-stimulating hormone from the pituitary gland, study of the properties of these autoantibodies confirmed the

Corresponding author: Duncan Dartrey Adams, MD, DSc, FRACP, research fields: medicine, endocrinology, genetics, autoimmune disease. E-mail: duncan.adams@xtra.co.nz.
Forbidden Clone theory by showing that they originate from single lymphocytes and show fine variation from patient to patient indicative of the random element in the mutations that produced them [5].

Graves' disease, a paradigm for autoimmune disease. In 1986, at the University of Pisa, Professor Aldo Pinchera led an International Symposium on Thyroid Autoimmunity at which Graves' disease was presented as a paradigm of autoimmune disease [6]. This came from the experimental advantages provided by the presence of iodine in thyroid hormone, the hormone receptor nature of the autoantigen and the control of thyroid activity by the pituitary gland.

\section{Genetics}

The complicated genetics of the autoimmune diseases was revealed by studies of families, including twins, which show involvement of multiple co-dominant genes with incomplete penetrance [7]. 
Vladutiu and Rose [8] discovered involvement of the major histocompatibility gene complex (MHC), which had been discovered in the field of surgical transplantation [9]. In man, a collection of genes on chromosome 6 code for peptides expressed on the surface of all nucleated cells. In the mouse a similar collection is on chromosome 17. Why does the MHC exist? Zinkernagel and Dogherty [10] discovered this by showing that cells infected by a virus extrude viral peptides on to their surface histocompatibility antigens, which they alter, causing the cell to be destroyed by the circulating cytotoxic $\mathrm{T}$ cell clones constantly checking for virus infection. The explosive speed of viral replication makes this mechanism necessary to prevent swift death of the virus-infected animal [11].

Building on the Bielschowsky's discovery of autoimmune anaemia in their NZB/BL strain of inbred mice, Howie and Helyer discovered that F1 hybrids of this strain with the healthy NZW strain unexpectedly develop the autoimmune kidney disease, lupus nephritis [12]. The occurrence of this disease in a hybrid, both of whose parents lack it, shows that at least one gene from each of the two parental strains causes the lupus nephritis. Back-cross and linkage studies showed that three genes contribute to the lupus nephritis [13] and two to the autoimmune anaemia. The linkage studies show that these are not the immunoglobulin $\mathrm{V}$ genes that were expected. Two are in the neighbourhood of minor histocompatibility antigen genes, $\mathrm{Hh}$ and $\mathrm{H}-18$. Putting together the research fields of autoimmunity and transplantation, Adams and Knight arrived at the $\mathbf{H}$ Gene Theory of inheritance of autoimmune disease [14]. This states that histocompatibility antigen genes, major, minor and $\mathrm{HY}$ (the male sex antigen), together with the $\mathrm{V}$ (variable region) genes coding for antigen receptors on $\mathrm{B}$ and $\mathrm{T}$ lymphocytes, are the germ-line immune response genes, the genes that influence the risk of autoimmune disease. The $\mathrm{H}$ Gene Theory received general acceptance and wide admiration when delivered by John Knight to a distinguished audience at a Ciba Foundation Symposium in London in 1982 [15].

\section{Microbial Triggers}

Autoimmune diseases are caused by malfunction of the immunity system, being consequences of infection by bacteria or viruses $[16,17]$.

\subsection{Rheumatic Carditis and Streptococci}

Before the advent of penicillin, rheumatic fever, with crippling or fatal lesions of the heart, was a frequent consequence of infection by $\beta$-haemolytic streptococci of Lancefield Group A. This was because of an antigenic similarity between a component of these streptococci and heart tissue, discovered by Kaplan and Meyeserian [18]. Today, with such infections therapeutically aborted by penicillin, rheumatic heart disease, once common, has virtually disappeared.

\subsection{Glomerulonephritis and Streptococci}

Post-infective glomerulonephritis follows infection by Group A streptococci of multiple $\mathrm{M}$ types. This disease is also less frequent due to use of antibiotics.

\subsection{Reactive Arthritis}

This has been observed after enteric infection with Shigella, Salmonella, Yersinia, Compylobacter and genital infection with Neisseria gonorrhea.

\subsection{Rheumatoid Arthritis (RA) and Proteus Mirabilis [19]}

Multiple studies over three decades have found high titres of antibodies against this bacterium in a total of 1375 RA patients, but not in other diseases or healthy controls in studies by independent groups in 15 different countries. There was no such elevation in antibodies against 27 other microbial agents. There is evidence that the upper urinary tract is the main site of Proteus infection in RA. 


\subsection{Ankylosing Spondylitis (AS) and Klebsiella [20]}

In worldwide studies involving 1330 AS patients and 1191 healthy controls, the AS patients showed significantly increased antibody titres to Klebsiella. There is evidence that the gut is the main site of Klebsiella infection in AS.

\subsection{Type 1 Diabetes and Coxsackie Virus}

Richter and Horowitz [21] present the considerable evidence that Coxsackie viruses, especially B4, may trigger type1 diabetes, including the discovery that there are shared regions of homology between the coxsackie virus protein PC-2 and the islet antigen GAD65.

\subsection{Systemic Sclerosis and Infections}

Randone et al. [22] describe the evidence suggesting that Parvovrus B19, Cytomegalovirus, Ebstein-Barr virus, Endogenous retrovirus, or Helicobacter pylori infection might trigger systemic sclerosis, where it has been postulated that fibroblast-stimulating forbidden clones, probably of $\mathrm{B}$ cell origin, are the immunological agent [7].

\subsection{Schizophrenia and Virus Infection}

Acute schizophrenia has been observed to follow upper respiratory tract virus infection. Knight et al. have assembled much evidence indicating that schizophrenia is an autoimmune disease caused by autoantibodies that react with neuronal receptors influencing the limbic system [23-25].

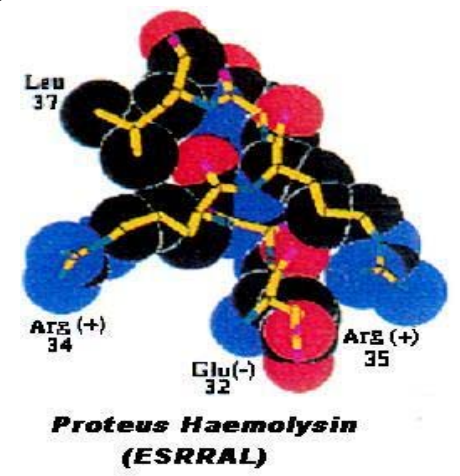

\section{Information from Sequencing Antigens on Triggering Bacteria}

\subsection{Basic Book}

Details of the development of the methods used for successful determination of the amino acid sequences of antigens on the autoimmune disease-triggering bacteria, Proteus mirabilis and Klebsiella pneumoniae, are recorded in the book, "Rheumatoid arthritis and Proteus” by Ebringer [26].

\subsection{Confirmation of the H Gene Theory}

This research provides experimental confirmation, at the molecular level, of the $\mathrm{H}$ Gene Theory of the inheritance of the autoimmune diseases, described above, in confirming the speculated presence of multiple antigens on triggering bacteria and alternative clonal development causing development of the forbidden clones that cause the associated autoimmune diseases.

\subsection{How HLA-DR1/4 Predisposes to Rheumatoid} Arthritis [27]

Fig. 1 shows space-filling models of the amino acid sequences of the histocompatibility antigen, HLA-DR1/4 and the Proteus mirabilis haemolysin antigen. Close structural similarity is apparent. This means the immune tolerance imposed by the histocompatibility antigen will extend to this Proteus antigen, preventing immune reaction with it.

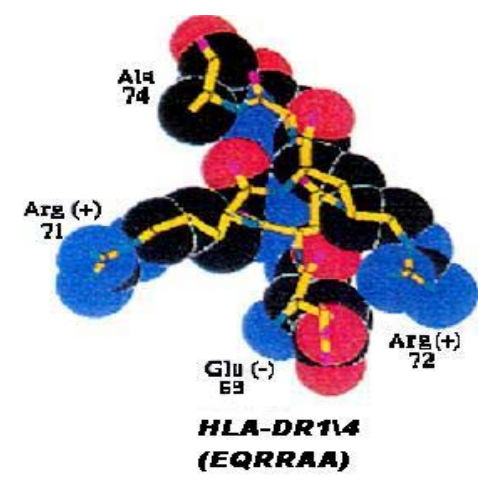

Fig. 1 Molecular similarity between histocompatibility antigen HLA-DR1/4 and Proteus haemolysin, preventing immune reaction against this bacterial antigen. 
Fig. 2 shows space-filling models of the amino acid sequences of the Proteus mirabilis urease antigen and Type 11 collagen, an autoantigen attacked in rheumatoid arthritis. The urease antigen is completely different from HLA-DR1/4, so will not be protected from immune reaction, being free to stimulate development of a forbidden clone reacting with the closely similar Type 11 collagen molecule, an autoantigen attacked in rheumatoid arthritis.

\subsection{How HLA-B27 Predisposes to Ankylosing Spondylitis [28]}

Fig. 3 shows space-filling models of the amino acid sequences of the histocompatibility antigen, HLA-B27 and two antigenic peptides on the bacterium Klebsiella pneumoniae. The Klebsiella nitrogenase antigen closely resembles HLA-B27, so will be covered by the tolerance induced by HLA-B27, but the bacterium's pullanase peptide is different and able to stimulate development of a forbidden clone, attacking the spine to cause causing ankylosing spondylitis.

\section{Prophylaxis of Autoimmune Diseases}

\subsection{The Poliomyelitis Virus Epidemics}

A New Zealand example occurred in 1938, reported in the press and observed by Adams, trapped in a boarding school at Masterton. An epidemic of leg

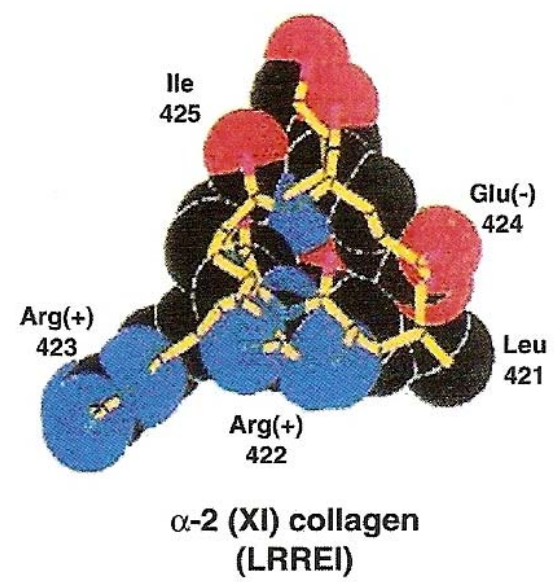

paralyses occurred in Christchurch and spread progressively north, from town to town, to Picton, Wellington, Featherstone, then Carterton, the town next to Masterton, engendering great fear. Then, the boy in the bed next to Adams, complained of a stiff neck, was taken away, and reported to have polio. Six months later he returned with a paralysed leg. Adams and his 30 school-mates were all unaffected.

\subsection{Paralysis a Rare Autoimmune Complication of Universal Virus Infection}

We postulate that the leg paralyses of poliomyelitis were a rare autoimmune complication of virtually universal virus infection, the paralyses probably caused by forbidden clones of cytotoxic T cells which attacked anterior horn neurons, hence the Pathologists' appropriate name, “acute anterior poliomyelitis”.

5.3 The Lead in Prophylaxis Given by the Polio Vaccines

The Salk (killed) and Sabin (attenuated) polio vaccines have both been brilliantly successful in preventing the polio leg paralyses. This exemplifies how autoimmune diseases in general, can be prevented by finding and vaccinating against their microbial triggers.

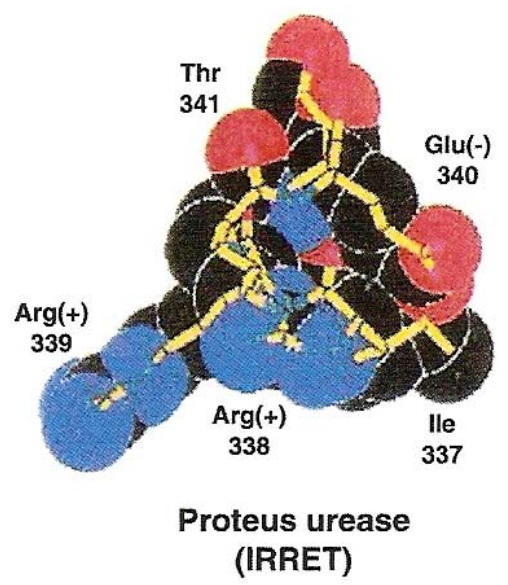

Fig. 2 Molecular dissimilarity of Proteus urease with HLA-DR1/4, allowing immune reaction with this bacterial antigen, which resembles Type $\mathrm{X} 1$ collagen, an autoantigen attacked in rheumatoid arthritis. 


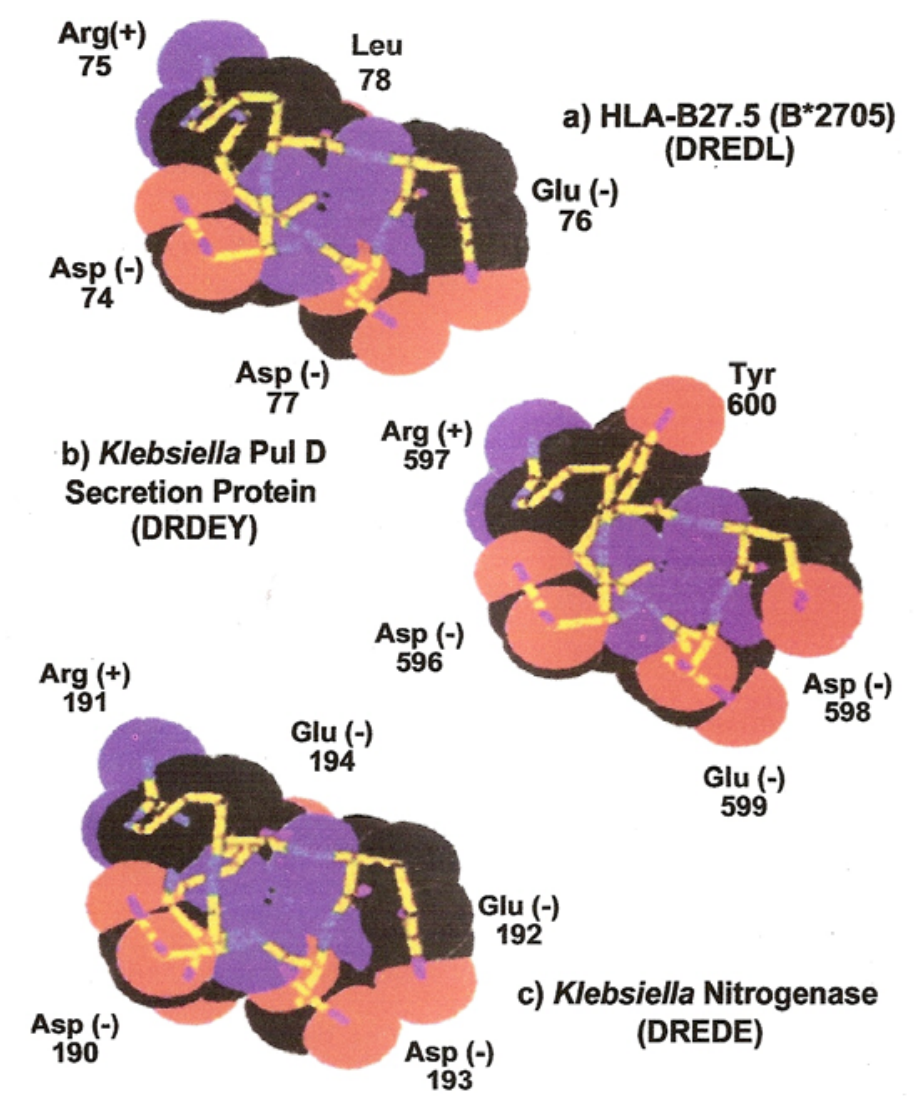

Fig. 3 Molecular similarity between HLA-B27 and the nitrogenase reductase peptide of Klebsiella, preventing immune reaction, and dissimilarity with the pullanase peptide (Pul D), of Klebsiella. This explains how HLA-B27 increases the risk of ankylosing spondylitis 69-fold, discovered by Terasaki.

\subsection{Finding Microbial Triggers}

Ebringer has succeeded in this with rheumatoid arthritis and ankylosing spondylitis. He has pioneered this new field of medical research, developing a whole new technology that needs to be copied in other disease, especially schizophrenia. Systematic studies of autoimmune diseases, with collaboration between clinicians and microbiologists, are needed. The American Academy of Microbiology would be an ideal organization for doing the research needed to provide this urgently-needed knowledge.

\section{Discussion}

Some disease associations are cross-tissue autoimmunity, for example the eye proptosis of Graves' disease, caused by variants of the thyroid-stimulating autoantibodies that react with receptors on orbital fat cells [5], and diabetic retinopathy [29], probably caused by destruction of retinal pericyte cells by antigenic variants of the $\mathrm{T}$ cell forbidden clones that destroy the pancreatic islet $\beta$ cells to cause Type 1 diabetes

Many autoimmune diseases, such as Graves' disease, already have satisfactory therapy.

Immunotherapy, by radiological or chemical immune ablation with immune reconstitution by autologous bone marrow cells, pioneered by Tyndall [30], can be used to save the lives of patients with dangerous autoimmune diseases, such as systemic scleroderma [31].

Selective destruction of forbidden clones could be achieved by isolating their autoantigen (such as the TSH receptor of Graves' disease, cloned by Vassart and Dumont) [32] and attaching it to a cytotoxic moiety, such as bungarotoxin or 131I iodine (emitting 
short-range beta particles), then admistering the molecular complex intravenously to destroy the pathogenic clones of plasma cells.

When monoclonal antibody technology was discovered, it was mistakenly assumed that this would provide cures for the autoimmune diseases, a notion greatly encouraged by the drug companies. Struggling to help his patients get benefit from use of Rituximab, Dreyfus [33] envisages major progress from anti-viral therapies and ultimately virus vaccines.

Prevention is better than cure, so finding and countering antigenic triggers of autoimmune diseases is the ideal. Recognition of the universality of microbial triggers of autoimmune diseases is a major advance, in showing how the diseases can be prevented by finding and vaccinating against their triggers. Ebringer has led the way by discovering two triggers and developing the technology for finding others.

\section{Acknowledgements}

Dr Adams is indebted to Pro-Vice Chancellor Peter Crampton for administrative support and to $\mathrm{Dr}$ Christopher Adams for electronic assistance.

\section{References}

[1] D. D. Adams, J. G. Knight and A. Ebringer, Autoimmune diseases: Solution of the environmental, immunological and genetic components with principles for immunotherapy and transplantation, Autoimmun Rev. 9 (2010) 525-530.

[2] N. K. Jerne, The selection theory of antibody formation, Proc Nat Acad Sci USA 41 (1955) 849-853.

[3] F. M. Burnet, The clonal selection theory of acquired immunity, London: Cambridge University Press, 1959.

[4] F. M. Burnet, Autoimmune disease, BMJ 2 (1959) 645-650, 720-725.

[5] D. D. Adams, Thyroid-stimulating autoantibodies, Vitamins and Hormones 38 (1980) 119-203.

[6] D. D. Adams, A. Knight, J. G. Knight, P. Laing, Graves' disease: A paradigm for autoimmunity, in: A. Pinchera, S. H. Ingbar, J. M. McKenzie, G. F. Fenzi (Eds.), Thyroid Autoimmunity, New York: Plenum, 1987, pp. 1-10.

[7] D. D. Adams and J. G. Knight, Principles of autoimmune disease: Pathogenesis, genetics and specific immunotherapy, J Clin Lab Immunol 52 (2003) 1-22.
[8] A. O. Vladutiu and N. R. Rose, Autoimmune murine thyroiditis: Relation to histocompatibility type, Science 174 (1971) 1137-1138.

[9] H. Auchincloss, M. Sykes and D. H. Sachs, Transplantation immunology, in: W. E. Paul (Ed.), Fundamental Immunology (4th ed.), Philadelphia: Lippincott-Raven, 1999, pp. 1175-1235.

[10] R. M. Zinkernagel and P. C. Doherty, Restriction of in vitro $\mathrm{T}$ cell-mediated cytotoxicity in lymphocytic choriomeningitis within a syngeneic or semi-allogeneic system, Nature 248 (1947) 701-702.

[11] D. D. Adams, Protection from autoimmune disease as the third function of the major histocompatibility gene complex, Lancet ii (1987) 245-249.

[12] J. B. Howie and B. J. Helyer, The immunology and pathology of NZB mice, Adv Immunol. 9 (1968) 215-266.

[13] J. G. Knight and Adams D. D., Three genes for lupus nephritis in NZB x NZW mice, J. Exp Med 147 (1978) 1653-1660.

[14] D. D. Adams and J. G. Knight, The H gene theory of inherited autoimmune disease, Lancet i (1980) 396-398.

[15] J.G. Knight and D. D. Adams, The genetic basis of autoimmune disease, Ciba Foundation Symposium 90 (1982) 35-56.

[16] A. Doria, P. Sarzi-Puttini and Y. Shoenfeld, Infections, rheumatism and autoimmunity: The conflicting relationship between humans and their environment, Autoimmun Rev 8 (2008) 1-4.

[17] A. Doria, S. Zampieri and P. Sarzi-Puttni, Exploring the complex relationship between infection and autoimmunity, Autoimmun Rev 8 (2008) 89-91.

[18] M. H. Kaplan and M. Meyeserian, An immunological cross-reaction between Group A streptococcal cells and human heart tissue, Lancet i (1962) 706-710.

[19] A. Ebringer, T. Rashid and C. Wilson, Rheumatoid arthritis, Proteus, anti-CCP antibodies and Karl Popper, Autoimmun Rev 9 (2010) 216-223.

[20] A. Ebringer, T. Rashid, T. Wilson, T. Ptaszynska and M. Fielder, Ankylosing spondylitis, HLA-B27, and Klebsiella - An overview: Proposal for early diagnosis and treatment, Curr Rheumatol Rev 2 (2006) 55-68.

[21] M. J. Richter and M. S. Horowitz, Coxsachie virus infection as an environmental factor in the etiology of type 1 diabetes, Autoimmun Rev. 8 (2008) 611-615.

[22] S. B. Randone, S. Guiducci and M. M. Cerinic, Systemic sclerosis and infection, Autoimmun Rev 8 (2008) 36-40.

[23] J. G. Knight, A Knight and C. B. Pert, Is schizophrenia a virally-triggered anti-receptor autoimmune disease? in: $\mathrm{H}$. Helmchen, F. A. Henn (Eds.), Biological Perspectives of Schizophrenia, New York: Wiley, 1987, pp. 107-127. 
[24] P. Laing, J. G. Knight, J. M. Hill, A. G. Harris and R. G. Webster et al., Influenza viruses induce autoantibodies to a brain-specific 37-kDa protein in rabbit, Proc Natl Acad Sci USA 86 (1989) 1998-2002.

[25] J. G. Knight, D. B. Menkies, J. Highton and D. D. Adams, Rationale for a trial of immunosuppressive therapy in acute schizophrenia, Mol Psychiatry 12 (2007) 424-431.

[26] A. Ebringer, Rheumatoid Arthritis and Proteus, London: Springer, 2011.

[27] C. Wilson, A. Ebringer, K. Ahmadi, J. Wrigglesworth, H. Tiwana, M. Fielder, A. Binder, C. Ettelaie, P. Cunningham, C. Joannou and S. Bansal, Shared amino acid sequences between major histocompatibility complex class II glycoproteins, type XI collagen and Proteus mirabilis in rheumatoid arthritis, Annals of the Rheumatic Diseases 54 (1995) 216-220.

[28] A. Ebringer, T. Rashid, C. Wilson, T. Ptazynska and M. Fielder, Ankylosing spondylitis, HLA-B27 and Klebsiella pneumoniae - An overview proposed for early diagnosis and treatment, Curr Rheumatol Rev 2 (2006) 55-68.

[29] D. D. Adams, Autoimmune destruction of pericytes as the cause of diabetic retinopathy, Clin Ophthalmol 2 (2008) 295-298.

[30] D. Forge, J. Passweg and J. M. Van Laar et al., Autologous stem cell transplantation in the treatment of systemic sclerosis: Report from the EBMT/EULAR Registry. Ann Rheum Dis 63 (2004) 974-981.

[31] H. Englert, C. Katelaris, N. McGill et al. Grape-saltana sign represents a favourable response to aggressive treatment of early diffuse systemic scleroderma. Intern Med J 35 (2005) 436-437.

[32] J. Peret, M. Ludgate and F. Libert et al., Stable expression of the human TSH receptor in $\mathrm{CHO}$ cells and characterization of differentially expressing clones. Biochem Biophys Res Commun 171 (1990) 1044-1050.

[33] D. H. Deyfus. Autoimmune disease: A role for new anti-viral therapies? Autoimmun Rev 11 (2011) 88-97. 